\title{
Associative Learning Drives the Formation of Silent Synapses in Neuronal Ensembles of the Nucleus Accumbens
}

\author{
Leslie R. Whitaker, Paulo E. Carneiro de Oliveira, Kylie B. McPherson, Rebecca V. Fallon, \\ Cleopatra S. Planeta, Antonello Bonci, and Bruce T. Hope
}

\begin{abstract}
BACKGROUND: Learned associations between environmental stimuli and rewards play a critical role in addiction. Associative learning requires alterations in sparsely distributed populations of strongly activated neurons, or neuronal ensembles. Until recently, assessment of functional alterations underlying learned behavior was restricted to global neuroadaptations in a particular brain area or cell type, rendering it impossible to identify neuronal ensembles critically involved in learned behavior.

METHODS: We used Fos-GFP transgenic mice that contained a transgene with a Fos promoter driving expression of green fluorescent protein (GFP) to detect neurons that were strongly activated during associative learning, in this case, context-independent and context-specific cocaine-induced locomotor sensitization. Whole-cell electrophysiological recordings were used to assess synaptic alterations in specifically activated GFP-positive (GFP + ) neurons compared with surrounding nonactivated GFP-negative (GFP-) neurons 90 min after the sensitized locomotor response.

RESULTS: After context-independent cocaine sensitization, cocaine-induced locomotion was equally sensitized by repeated cocaine injections in two different sensitization contexts. Correspondingly, silent synapses in these mice were induced in GFP + neurons, but not GFP - neurons, after sensitization in both of these contexts. After contextspecific cocaine sensitization, cocaine-induced locomotion was sensitized exclusively in mice trained and tested in the same context (paired group), but not in mice that were trained in one context and then tested in a different context (unpaired group). Silent synapses increased in GFP+ neurons, but not in GFP- neurons from mice in the paired group, but not from mice in the unpaired group.
\end{abstract}

CONCLUSIONS: Our results indicate that silent synapses are formed only in neuronal ensembles of the nucleus accumbens shell that are related to associative learning.

Keywords: Addiction, Electrophysiology, Glutamate, Memory, Psychostimulant, Transgenic

http://dx.doi.org/10.1016/j.biopsych.2015.08.006

Learned associations between drugs of abuse and environment stimuli play a critical role in drug addiction. One of the key aspects of these drug-related memories is that one specific stimulus can induce a conditioned drug behavior such as relapse, whereas other unrelated stimuli do not. The neurobiological mechanism that underlies this type of learning must encode these drug-related memories with a degree of resolution sufficient to discriminate between memories activated by different sets of stimuli. Recent studies support the hypothesis that specific patterns of sparsely distributed Fosexpressing neurons act together as neuronal ensembles that mediate addiction-related learned behaviors (1-6), including context-specific expression of reinstatement of drug seeking $(1,2)$, incubation of drug craving (3), and context-specific locomotor sensitization (4). These Fos-expressing neuronal ensembles are formed by only a few neurons $(\sim 2 \%-12 \%)$ that are selected by the relevant cues. The enormous number of possible permutations of these neuronal ensembles provides sufficiently high resolution to discriminate between different associative memories. Selective alterations within these neurons are likely to play a key role in learning and maintenance of these drug-related memories.

Synaptic plasticity is the key candidate neural mechanism for encoding learning and memory processes. Many years of research have shown that exposure to cocaine and other drugs of abuse can induce synaptic alterations in rewardrelated brain areas such as the nucleus accumbens and ventral tegmental area $(7-11)$. These neuroadaptations include alterations in the balance of alpha-amino-3-hydroxy-5-methyl4-isoxazole propionic acid (AMPA) receptor-mediated and $N$-methyl-D-aspartate receptor-mediated transmission $(7,9)$, changes in AMPA receptor subunit composition $(12,13)$, alterations in synaptic plasticity $(11,14)$, and the development of silent synapses $(15,16)$. Synaptic alterations in 
addiction-relevant brain areas have also been shown to play an important role in the development and persistence of behavioral models in addiction research (17-19). However, these synaptic alterations are observed in whole-brain areas or in particular cell types regardless of their neural activity during the learned behaviors. Although these global alterations can play important general roles in learning and maintenance of memories, other mechanisms are required to encode the highresolution information in cue-specific memories and discriminate them from other memories stored in the same brain area. Based on previous studies from our laboratory and others, we hypothesize that unique synaptic alterations induced within drug cue-activated Fos-expressing neuronal ensembles play a critical role in the expression of many learned associations in conditioned drug behaviors $(5,6)$.

We used Fos-GFP transgenic mice that contained a transgene with a Fos promoter driving expression of green fluorescent protein (GFP) to detect neurons after context-specific sensitization of cocaine-induced locomotion sensitization (20). The small proportion of GFP-expressing neurons that was strongly activated during sensitized cocaine-induced locomotion exhibited large percentage increases of silent synapses and related synaptic alterations that were not present in most surrounding GFP - neurons that were less activated during behavior. We hypothesized that the emergence of silent synapses might represent a cellular mechanism contributing to the development of learned associations, but we were unable to determine this because mice were trained and tested in the same context. Under these circumstances, the development of silent synapses could result from a compensatory response of neurons that were particularly strongly activated by repeated exposure to cocaine with no role in associative learning. To address whether silent synapses are induced in a context-specific manner, we examined synaptic alterations in GFP + and GFP - neuronal populations after two different forms of cocaine-induced locomotor sensitization: 1) context-independent form of sensitization in which animals displayed a sensitized locomotor response regardless of their previous training context and 2) context-specific form of sensitization in which mice were able to distinguish between two different contexts and displayed a sensitized locomotor response only when tested in the context in which they were trained.

\section{METHODS AND MATERIALS}

\section{Animals}

We used 163 male and female Fos-GFP transgenic mice in our experiments. These mice were initially obtained in 2008 from Alison Barth at Carnegie Mellon University. We bred male heterozygous Fos-GFP mice with female wild-type C57BL/6 mice (Charles Rivers Laboratories International, Inc., Wilmington, Massachusetts) for $>15$ generations at the Intramural Research Program facilities of the National Institute on Drug Abuse. An additional 24 male wild-type C57BL/6 mice were obtained from Charles Rivers Laboratories. Mice were maintained in a temperature-controlled and humidity-controlled facility on a 12-hour light-dark cycle. Mice were separated and individually housed with ad libitum food and water for 3-5 days before training. All experiments were conducted during the light phase. Animal protocols were approved by the Animal Care and Use Committee of the National Institute on Drug Abuse Intramural Research Program and were carried out according to U.S. National Institutes of Health Guidelines.

\section{Behavioral Procedures}

Context-Independent Sensitization. Male and female Fos-GFP mice ( $n=47$ ) were habituated three times once daily for 60 minutes each day in locomotor activity chambers ( $43 \times 43 \mathrm{~cm}$, Med Associates, Inc., St. Albans, Vermont) and then divided randomly into two groups with approximately equal numbers of male and female mice. Mice in the paired group were injected once daily for 5 days with cocaine $(15 \mathrm{mg} / \mathrm{kg}$ intraperitoneal [i.p.]) or saline $(10 \mu \mathrm{L} / \mathrm{g})$ in the locomotor activity chamber (Context A). Mice in the unpaired group were injected once daily for 5 days with cocaine $(15 \mathrm{mg} / \mathrm{kg}$ i.p.) or saline (10 $\mu \mathrm{L} / \mathrm{g})$ in a different context (Context B-Independent), which was circular and contained a grid floor. On test day, after 6-11 days in their home cages, all mice were given a single injection of cocaine (20 mg/kg i.p.) in Context $A$ and perfused 90 minutes later.

Context-Specific Sensitization. Male and female Fos-GFP mice $(n=116)$ were divided randomly into two groups with approximately equal numbers of male and female mice. Mice in the paired group were injected once daily for 5 days with cocaine (15 mg/kg i.p.) or saline $(10 \mu \mathrm{L} / \mathrm{g})$ in the locomotor activity chamber (Context A). Mice in the unpaired group were injected once daily for 5 days with cocaine $(15 \mathrm{mg} / \mathrm{kg}$ i.p.) or saline $(10 \mu \mathrm{L} / \mathrm{g})$ in a different context (Context B-Specific), which was a round bowl with bedding and a toy; lights were dimmed and music (No Doubt, "Tragic Kingdom") was played continuously in this context. On test day, after 6-11 days in their home cages, all mice were given a single injection of cocaine (20 mg/kg i.p.) in Context $A$ and perfused 90 minutes later. In a separate experiment, 24 male C57BL/6 mice received identical training, but on test day, half the mice received cocaine injections (20 mg/kg), and half received saline injections $(10 \mu \mathrm{L} / \mathrm{g})$.

\section{Immunohistochemistry}

Fos-GFP mice were transcardially perfused 90 minutes after the final injection of cocaine on test day with $4 \%$ paraformaldehyde. Fos immunohistochemistry and Fos and neuronal nuclear antigen (NeuN) double-labeling were performed as previously described (4). For detailed information, see the Supplement.

\section{Ex Vivo Brain Slice Electrophysiology}

On test day, Fos-GFP mice were deeply anesthetized with isoflurane (60-90 s) 90 minutes after cocaine injections and transcardially perfused with ice-cold cutting solution. Coronal brain slices containing nucleus accumbens shell were prepared, and whole-cell voltage-clamp recordings were performed in GFP+ and GFP- medium spiny neurons of the nucleus accumbens shell as previously described (20). Spontaneous excitatory postsynaptic current (EPSC) data were collected using WinEDR software (courtesy of J. Dempster, University of Strathclyde, Glasgow, United Kingdom) and 
analyzed using Mini Analysis (Synaptosoft, Inc., Decatur, Georgia). Minimal stimulation assays were performed as previously described $(16,20)$. For additional information, see the Supplement.

\section{Statistics}

Group data are presented as mean \pm SEM. For detailed information, see the Supplement.

\section{RESULTS}

\section{Context-Independent Sensitization}

We used a sensitization procedure (Figure 1A) similar to the sensitization procedure in our previous study (20) to assess cocaine-induced locomotor sensitization in Fos-GFP mice. Mice in the paired group received repeated injections in the locomotor activity chamber (Context A) during training and were injected in the same Context $A$ on test day. Mice in the unpaired group received repeated injections in Context B-Independent during training and were injected in Context $A$ on test day. Using two-way analysis of variance (ANOVA), we found that repeated cocaine injections induced robust locomotor sensitization, regardless of training context (for repeated drug $\left[F_{1,43}=30.69, p<.0001\right]$; not significant for training context or interaction) (Figure 1B, C). When locomotor activity was assessed over time, cocaine-induced locomotion was significantly enhanced by previous repeated cocaine injections (for repeated drug $\left[F_{1,46}=19.51, p<.001\right]$ ) after test injections. Overall, sensitization was context-independent using these experimental conditions.

\section{Synaptic Alterations in Activated Neuronal} Ensembles After Context-Independent Sensitization

Coronal slices for slice electrophysiology were obtained 90 minutes after cocaine test injections from the mice that were repeatedly injected with cocaine ( $n=8-10$ cells from $6-9$ mice/group; $n=22$ mice in total), and synaptic alterations in GFP + and GFP - neurons of the nucleus accumbens shell were compared (Figure 4A). We began by assessing the percentage of silent synapses using the minimal stimulation assay (for additional information see the Supplement). Example experiments and traces are shown in Figure 4B. In mice that received repeated cocaine injections, two-way ANOVA
A

Context-independent sensitization

$\begin{array}{lll}\text { Habituation } & \begin{array}{c}\text { Repeated injections } \\ \text { of saline or cocaine }\end{array} \text { Home cage } \\ \text { Test day } \\ \text { cocaine }\end{array}$

B

Cocaine-induced locomotion

Repeated injections

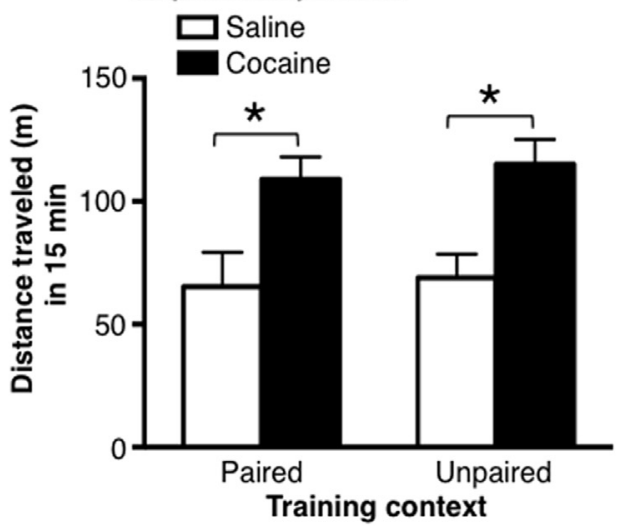

C

Test day locomotion

Repeated injections

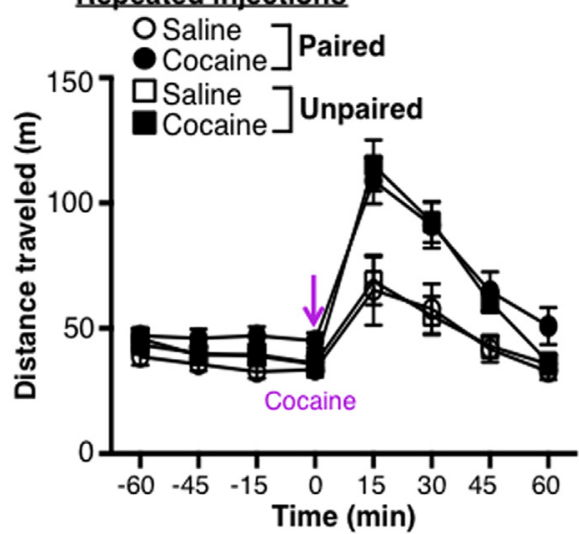

Figure 1. Context-independent sensitization of cocaine-induced locomotor activity. (A) Timeline for contextindependent sensitization experiment. All mice were habituated first for three times once daily for 60 minutes each in Context A. Mice in the paired group were injected five times once daily with saline or cocaine in the same Context $\mathrm{A}$, and mice in the unpaired group were injected five times once daily with saline or cocaine in Context B-Independent (Context B-Ind). After 1 week in the home cage, all mice were habituated for 60 minutes before test injections of $20 \mathrm{mg} / \mathrm{kg}$ cocaine in Context A. (B) Cocaine-induced locomotion during the first 15 minutes on test day ( $n=8-14$ mice/group). Repeated cocaine injections increased cocaine-induced locomotion $\left({ }^{\star} p<.0001\right)$, but this was not altered by the test context. (C) Time course of locomotion on test day shown in 15minute time intervals. 
indicated that the percentage of silent synapses was higher in GFP + neurons than in GFP - neurons, regardless of training context (for GFP expression $\left[F_{1,31}=70.43, p<.0001\right.$ ]; not significant for training context or interaction) (Figure 4C). In mice that received repeated saline injections, a $t$ test indicated that the percentage of silent synapses was not different between GFP + and GFP - neurons $\left(t_{15}=.79, p=.44\right.$, $n=8-9$ cells/group, GFP $-=6.52 \pm 3.06$, GFP $+=9.83 \pm$ 2.87 with paired/unpaired groups collapsed together). Under these experimental conditions, training context had no effect on development of silent synapses after cocaine-induced locomotor sensitization.

We also compared spontaneous EPSCs in GFP+ and GFP - neurons from paired and unpaired mice (example traces are shown in Figure 5A). Two-way ANOVA indicated a significantly lower frequency of spontaneous EPSCs in GFP+ than in GFP- neurons (for GFP expression $\left[F_{1,38}=15.75\right.$, $p<.001$ ]; not significant for training context or interaction). The amplitude of spontaneous EPSCs was not different between GFP + and GFP - neurons in paired or unpaired mice (Figure 5B). We also performed an alternative analysis of cumulative probability of spontaneous EPSC frequency and amplitude data (Figure 5C). Using the Kolmogorov-Smirnov test, we determined that there were significant differences in the cumulative probability of spontaneous EPSC frequencies between GFP + and GFP - neurons from paired $(p<.0001)$ and unpaired $(p<.05)$ groups, but no differences between groups for the cumulative probability of spontaneous EPSC amplitudes.

\section{Context-Specific Sensitization}

We used a different sensitization procedure to induce a context-specific form of sensitization in a separate group of Fos-GFP mice (Figure 2A). We removed the habituation period and made the alternative context (Context B-Specific) more distinct from the locomotor activity chamber (Context A). Context B-Specific was a large bowl containing bedding and a toy; lights in the room were dimmed and music was played throughout the session. On test day, mice were given a saline injection followed 60 minutes later by a test injection of cocaine $(20 \mathrm{mg} / \mathrm{kg})$ in Context A. Locomotor activity data were divided into 15-minute time intervals (Figure $2 \mathrm{~B}$ ) and analyzed using four-factor ANOVA, with training drug (cocaine, saline) and training context (A, B-Specific) as betweensubjects factors and test drug (cocaine, saline) and time ( -45 to 60 minutes) as within-subjects factors. There was a significant four-way interaction $\left(F_{3,138}=3.99, p<.01\right)$ and a significant three-way interaction between training drug, training context, and time $\left(F_{3,138}=7.33, p<.001\right)$. Post hoc analyses revealed a conditioned locomotion effect in the paired cocainetreated group after the saline test injection (time point -45 minutes $\left.\left[F_{1,49}=5.60\right]\right)$. The paired cocaine-treated group also displayed enhanced locomotion 15-30 minutes after the cocaine test injection (15 minutes $\left[F_{1,49}=7.31, p, .05\right], 30$ minutes $\left.\left[F_{1,49}=4.19, p<.05, p<.01\right]\right)$. We also analyzed locomotor data taken from the first 15 minutes after cocaine test injections (Figure 2C) using two-way ANOVA and found that there was a significant effect of training drug $\left(F_{1,49}=15.17\right.$, $p<.001)$ and a significant interaction $\left(F_{1,49}=5.24, p<.05\right)$. Bonferroni's post-test revealed a significant difference between mice trained with saline versus cocaine in the paired group only $(p<.0001)$. Mice expressed the learned behaviors contextspecific sensitization and conditioned locomotion.

\section{Synaptic Alterations in Activated Neuronal Ensembles After Context-Specific Sensitization}

Coronal slices for slice electrophysiology were obtained 90 minutes after cocaine test injections from mice after contextspecific sensitization ( $n=7-8$ cells from $5-7$ mice/group, $n=17$ mice in total), and synaptic alterations in GFP + and GFP - neurons of the nucleus accumbens shell of paired and unpaired mice were compared after context-specific sensitization. Example experiments and traces are shown in Figure $3 \mathrm{~B}$. In mice that received repeated cocaine injections, two-way ANOVA indicated significant main effects for GFP expression $\left(F_{1,25}=21.78, p<.0001\right)$ and training context $\left(F_{1,25}=16.94, p<.0001\right)$ and a significant interaction $\left(F_{1,25}=\right.$ 24.15, $p<.0001)$. Bonferroni's post hoc tests confirmed that the percentage of silent synapses was greater in GFP+ neurons than in GFP - neurons from paired mice, but not from unpaired mice (Figure $3 \mathrm{C}$ ). In mice that received repeated saline injections, a $t$ test indicated that the percentage of silent synapses was not different between GFP+ and GFP- neurons $\left(t_{8}=.41, p=.69 ; \mathrm{GFP}+, 9.21 \% \pm 4.10 \%\right.$; GFP-, $11.01 \% \pm$ $1.44 \%$ with paired/unpaired groups collapsed together).

We also examined spontaneous EPSCs in GFP+ and GFP - neurons from paired and unpaired mice (example traces are shown in Figure 6A). Two-way ANOVA of spontaneous EPSC frequency data indicated a significant interaction between GFP expression and training context $\left(F_{1,32}=8.22\right.$, $p<.01)$. Post hoc tests confirmed that spontaneous EPSC frequency was lower in GFP + neurons than in GFP - neurons from paired mice, but not in neurons from unpaired mice (Figure 6B). The amplitude of spontaneous EPSCs was not different between GFP + and GFP - neurons in paired or unpaired mice (Figure 6B). We also performed an alternative analysis of cumulative probability of spontaneous EPSC frequency and amplitude data (Figure 6C). Using the KolmogorovSmirnov test, we observed a rightward shift in cumulative probability of spontaneous EPSC frequency in the GFP+ neurons of the paired mice $(p<.0001)$. No differences were observed between groups for the cumulative probability of spontaneous EPSC amplitudes from paired or unpaired mice.

\section{Fos Immunohistochemistry}

We examined Fos expression in the nucleus accumbens shell of mice ( $n=6 /$ group, total $n=24$ ) that underwent contextspecific sensitization. Two-way ANOVA revealed no significant differences for main effects or interaction. An acute control experiment confirmed that Fos expression was greater after acute cocaine injections than after saline injections on test day for mice in paired and unpaired groups (for acute drug injection $\left[F_{1,12}=9.10, p=.011\right]$; not significant for Training context). For descriptive purposes only, we assessed the percentage of all neurons that expressed Fos by double-labeling for Fos and the neuronal marker NeuN (Figure 3A). We used an additional set of sections from mice that received cocaine test injections after context-specific sensitization. Approximately $5 \%$ of 
A Identification of GFP+ neurons in Fos-GFP mice
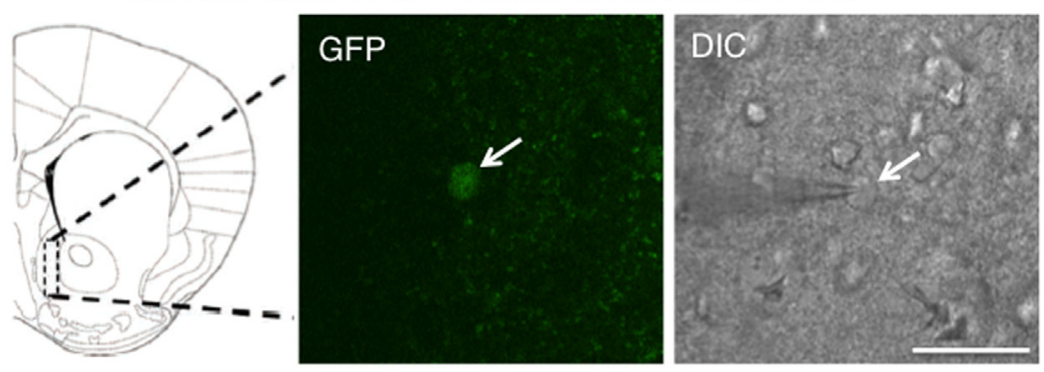

\section{B}

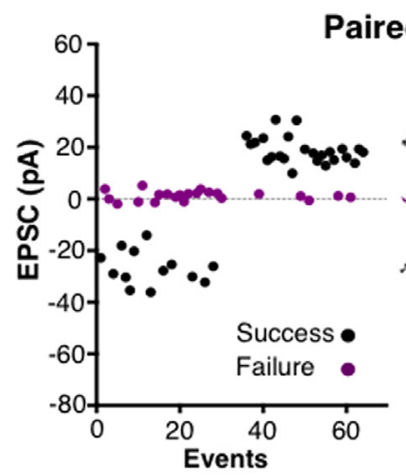

Representative experiments from GFP+ neurons
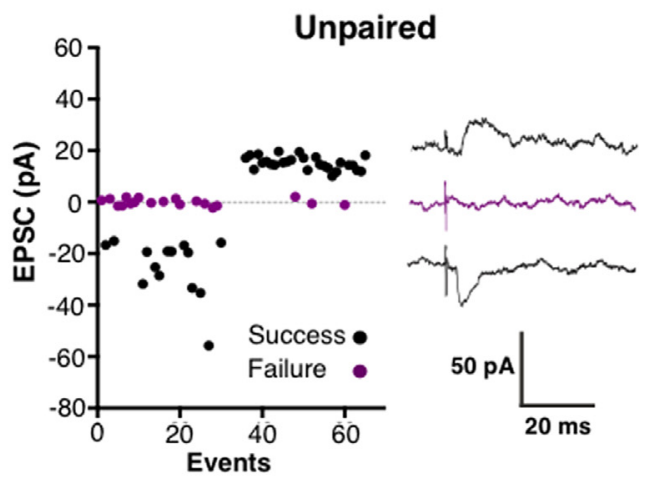

\section{Silent synapses following context-independent sensitization}

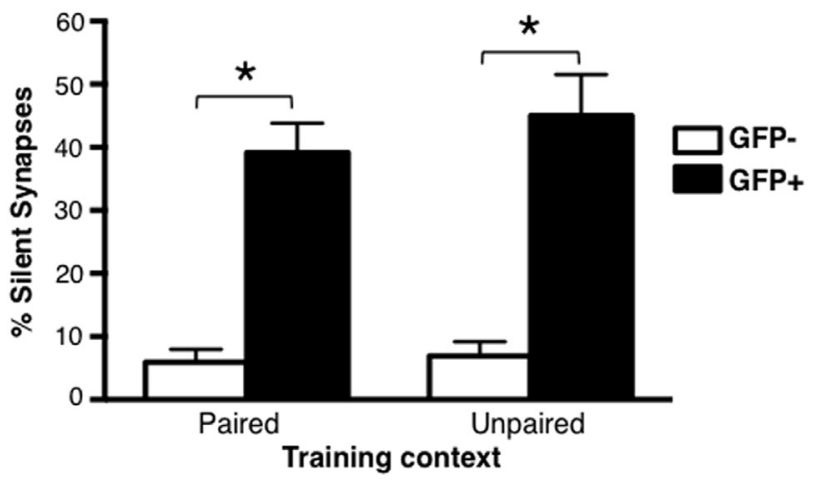

Figure 2. Synaptic alterations in green fluorescent protein-positive $(\mathrm{GFP}+)$ and green fluorescent protein-negative (GFP-) neurons after context-independent sensitization. (A) Activated GFP+ neurons (arrows) in the nucleus accumbens shell were identified by their endogenous GFP fluorescence using confocal microscopy (left panel) and patched using differential interference contrast optics (DIC) (right panel). Scale bar = $25 \mu \mathrm{m}$. (B) Representative minimal stimulation assay experiments of GFP + neurons from mice in the paired and unpaired groups. Dots in each graph represent consecutive excitatory postsynaptic currents (EPSC) from a single GFP+ neuron. Representative EPSC traces are shown to the right of the graphs. The top trace was recorded at a holding potential of $+40 \mathrm{mV}$ ( $N$-methyl-D-aspartate receptor- and alpha-amino-3-hydroxy-5-methyl-4isoxazole propionic acid receptormediated current). The middle trace (purple) is an example of a synaptic failure. The bottom trace was recorded at a holding potential of -80 mV (alpha-amino-3-hydroxy-5methyl-4-isoxazole propionic acid receptor-mediated current). (C) Percentage of silent synapses after context-independent sensitization ( $n=8-10$ cells from $6-9$ mice/ group). Silent synapses were more frequent in GFP+ neurons $\left({ }^{*} p<.0001\right)$ from mice in the paired and unpaired groups; training context had no significant effect.

NeuN-labeled neurons coexpressed Fos (paired, 5.07\% \pm $0.44 \%$; unpaired, $4.50 \% \pm 0.39 \%$ ). In the case of contextindependent sensitization, $2 \%-3 \%$ of NeuN-labeled neurons coexpressed Fos (4).

\section{DISCUSSION}

We examined whether silent synapses were induced in context-specific Fos-expressing neuronal ensembles in mouse nucleus accumbens shell 90 minutes after expression of the sensitized locomotor response. Mice that had undergone context-specific sensitization exhibited sensitized cocaine-induced locomotion when tested in the same context in which they were trained, but not when tested in a different context. Psychostimulant-induced locomotor sensitization has been reported for decades and may arise as a result of a pharmacologic effect of repeated drug exposure or the formation of an association between environmental stimuli and the rewarding effects of drug (21-23). Context-specific sensitization in mice required very distinct contexts because less separable differences in context did not produce contextspecific sensitization (24-26). For both forms of sensitization, silent synapses were increased exclusively in the small number of GFP+ neurons that were activated on test day and not in the surrounding GFP- neurons. In mice that had undergone context-specific sensitization, silent synapses were induced in GFP + neurons of mice in the paired group, but not in neurons of mice in the unpaired group, which corresponds 


\section{Context-independent sensitization}

\section{A} Paired

GFP-

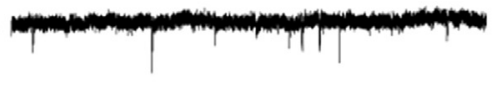

GFP-

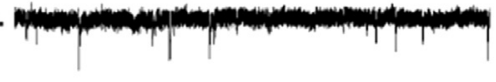

GFP+

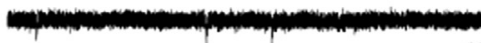
15 pAL

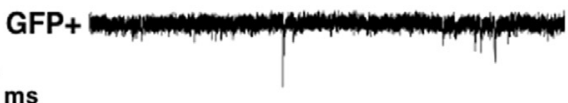

B

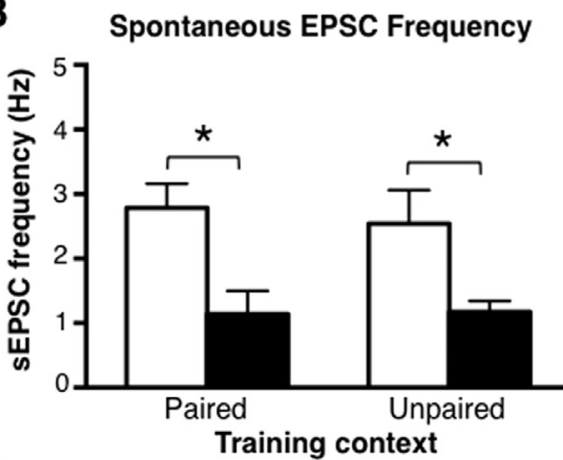

C

Paired: sEPSC Frequency

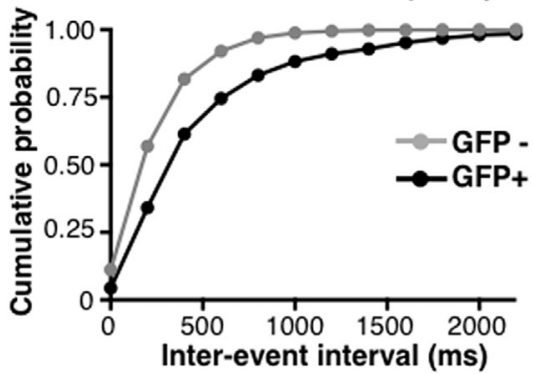

Unpaired: sEPSC Frequency

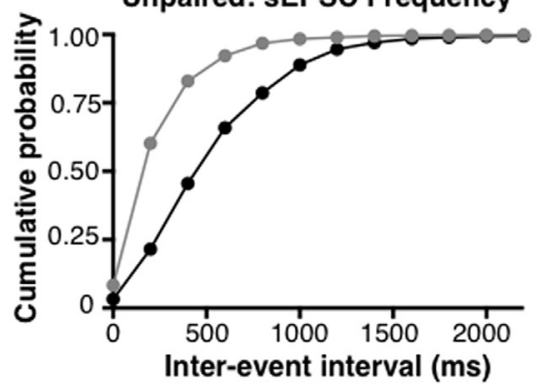

Spontaneous EPSC Amplitude

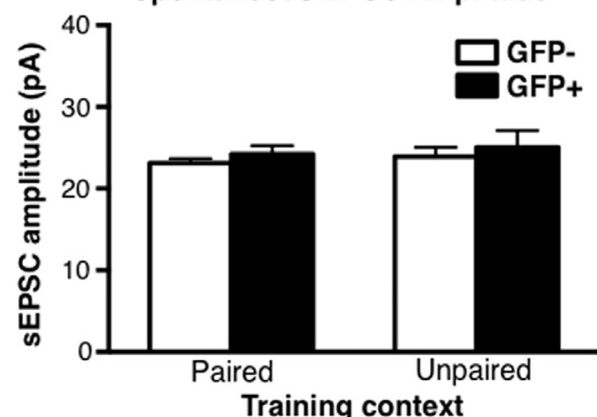

Paired: sEPSC Amplitude

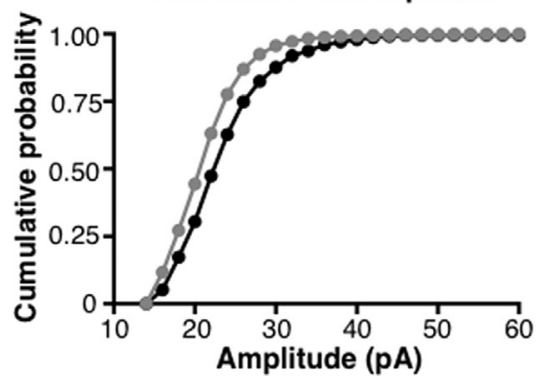

Unpaired: sEPSC Amplitude

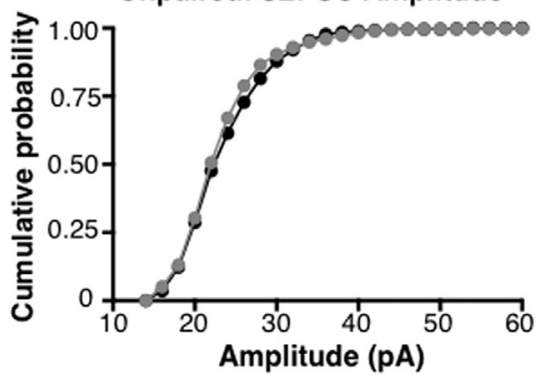

Figure 3. Quantal properties of spontaneous excitatory postsynaptic currents (sEPSCs) after context-independent sensitization. (A) Representative traces showing sEPSCs in green fluorescent protein-positive (GFP+) and green fluorescent protein-negative (GFP-) neurons from mice in the paired and unpaired groups. (B) sEPSCs frequencies (left) were significantly lower in GFP+ neurons (paired group, $n=10$ cells from 7 animals; unpaired group, $n=9$ cells from 7 animals) compared with GFPneurons (paired group, $n=12$ cells from 8 animals; unpaired group, $n=$ 10 cells from 7 animals) after contextindependent sensitization $\left({ }^{*} p<.001\right)$. sEPSC amplitudes (right) did not differ between groups. (C) Cumulative probability distributions of the same sEPSCs in (B). with context-specific sensitization of cocaine-induced locomotion.

Our context-independent sensitization procedure is similar to the protocol used in which we discovered more silent synapses in GFP + neurons after repeated cocaine injections (20). In this study, all mice were trained and tested in the same context, rendering it impossible to determine whether silent synapses in GFP + neurons arise as a result of pharmacologic effects of repeated cocaine experience or whether they are induced selectively in neurons determined by the drugassociated context. In the present context-independent experiment, silent synapses increased in the small proportion of GFP + neurons that were strongly activated on test day for paired and unpaired groups. Based on this experiment alone, either explanation is possible. However, an alternative explanation is that the mice in our context-independent sensitization experiment failed to distinguish between the two contexts, likely activating a similar neuronal ensemble in the nucleus accumbens shell and expressing sensitized locomotion in both contexts. The context-specific sensitization procedure allowed us to test this hypothesis. If the specific set of neurons that is repeatedly activated during training is determined by 
Context-specific sensitization

\begin{tabular}{|c|c|c|c|}
\hline \multirow[b]{2}{*}{ Paired } & $\begin{array}{l}\text { Repeated injections } \\
\text { of saline or cocaine }\end{array}$ & Home cage & $\begin{array}{l}\text { Test day } \\
\text { cocaine }\end{array}$ \\
\hline & 5 days & 7 days & 1 day \\
\hline & Context A & & Context A \\
\hline \multirow[t]{2}{*}{ Unpaired } & 5 days & 7 days & 1 day \\
\hline & Context B-Sp & & Context A \\
\hline
\end{tabular}

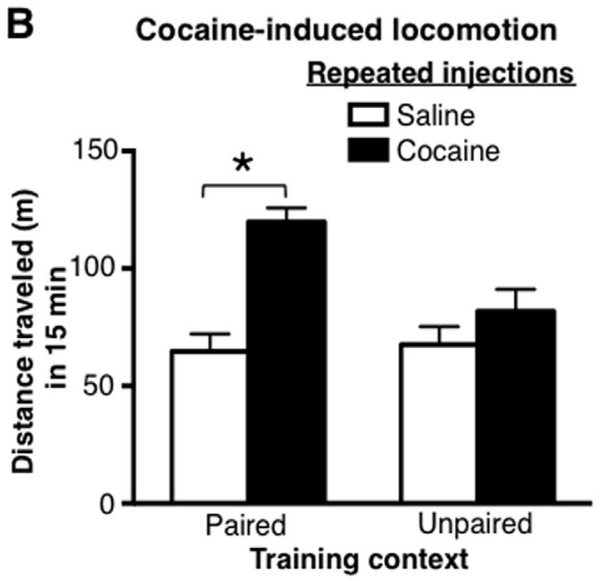

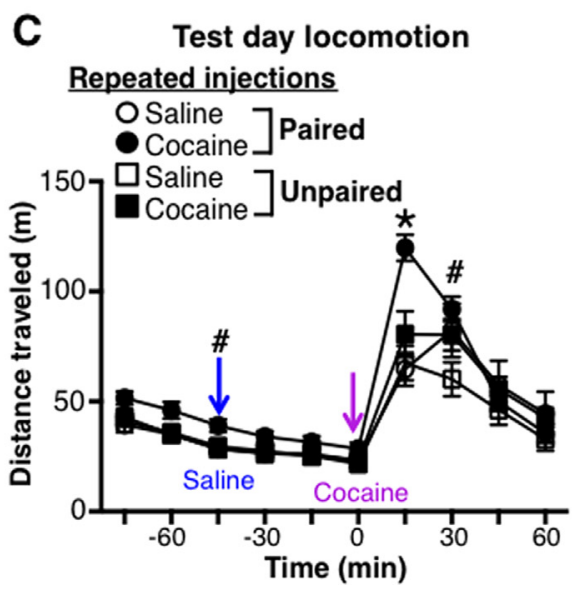

Figure 4. Context-specific sensitization of cocaine-induced locomotor activity. (A) Timeline for context-specific sensitization experiment. Mice in the paired group were injected five times once daily with saline or cocaine in Context $A$, and mice in the unpaired group were injected five times once daily with saline or cocaine in Context B-Specific (Context B-Sp). After 1 week in the home cage, all mice were habituated to the activity chamber for 30 minutes before test injections of saline and then cocaine in Context A. (B) Cocaine-induced locomotion during the first 15 minutes on test day $(n=$ 8-18 mice/ group). Repeated cocaine injections increased cocaine-induced locomotion $\left({ }^{*} p<.001\right)$ when tested in the same context where the mice received repeated injections (paired group), whereas repeated cocaine injections had no effect when tested in a different context where the mice received repeated injections (unpaired group). (C) Time course of locomotion on test day shown in 15-minute time intervals. Mice were habituated for 30 minutes and then given a saline injection followed by an injection of 20 $\mathrm{mg} / \mathrm{kg}$ of cocaine 60 minutes later. The paired group that received repeated cocaine injections displayed increased locomotion compared with the other groups for the first $15 \mathrm{~min}$ utes after saline injections and for the first 30 minutes after cocaine injections $\left({ }^{*} p<.01, \# p<.05\right)$. the context, changing the context on test day should activate a different set of neurons. Because silent synapses require repeated activation during sensitization (20), we should observe silent synapses in the paired group, but not in the unpaired group. We confirmed this in our context-specific sensitization experiment in which silent synapses were not induced in GFP + neurons from unpaired group mice. Silent synapses are induced only in context-selected neurons that were repeatedly activated during sensitization. The population of neurons expressing Fos after cocaine sensitization is small, representing $\sim 3 \%-5 \%$ of nucleus accumbens shell neurons (4). In vivo recording studies examined nucleus accumbens firing after cocaine self-administration and found that $\sim 25 \%$ of cells sampled show a phasic increase in firing after response-contingent cocaine administration $(27,28)$. Based on previous studies of Fos induction mechanisms, we hypothesize that Fos-expressing neurons in our study represent the most strongly activated subset of neurons undergoing increased phasic activity during repeated cocaine injections (5).

Context-specific selection of a small population of sparsely distributed neurons for silent synapse formation corresponds with the hypothesis that Fos-expressing neuronal ensembles encode memories to mediate learned behaviors $(5,6)$, which is based on Hebb's "cell assembly" hypothesis (29). A previous study from our laboratory had empirically shown that the same neuronal ensembles in the nucleus accumbens are reactivated after re-exposure to the same context after context-specific sensitization (30). We used double-labeling with deltaFosB immunohistochemistry to label neurons that were activated during repeated cocaine injections in one context and $c$-fos messenger RNA in situ hybridization to label neurons that were activated later in the same or different context; $87 \%$ of the same neurons were reactivated after reexposure to the same context. We also used the Daun02 inactivation procedure to inactivate Fos-expressing neuronal ensembles in the nucleus accumbens that were activated by cocaine injections in the drug-paired context and found reduced expression of sensitization when the rats were tested in the same drugpaired context 3 days later (4). This finding indicates that the same neuronal ensembles were reactivated by exposure to the same context. We have also shown that exposure to the same context reactivates the same neuronal ensembles in the ventromedial prefrontal cortex and nucleus accumbens to 
A

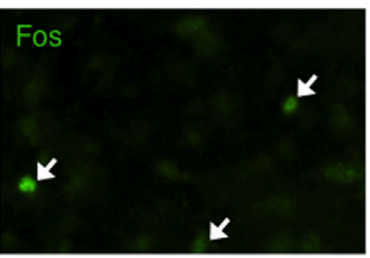

Fos+NeuN Double labeling
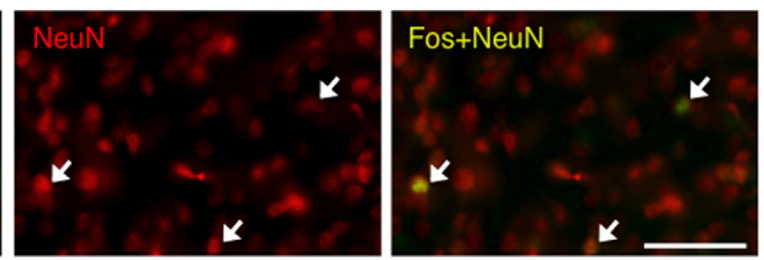

B

Representative experiments from GFP+ neurons
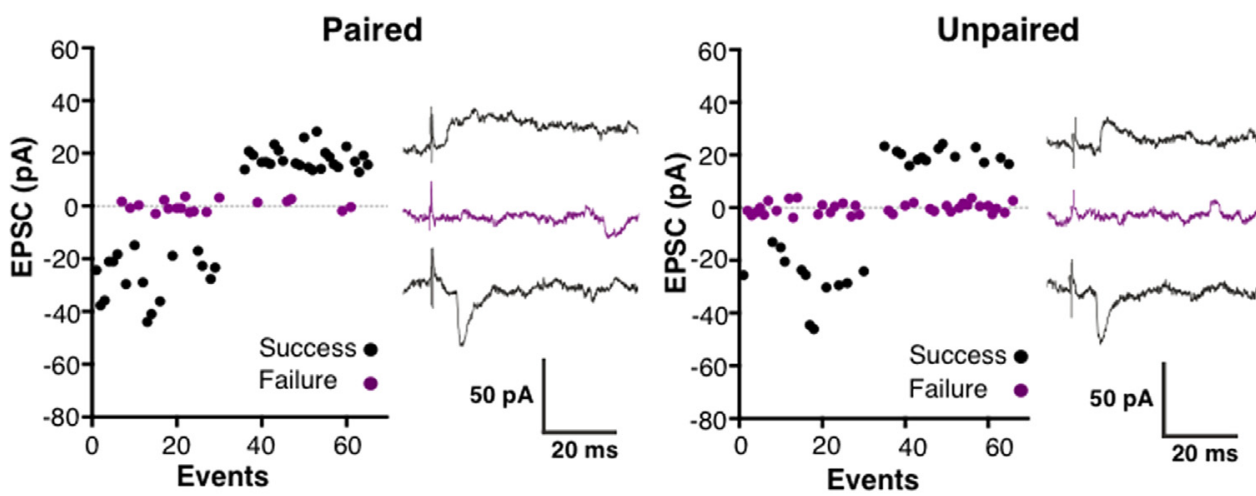

C

\section{Silent synapses following context-specific sensitization}

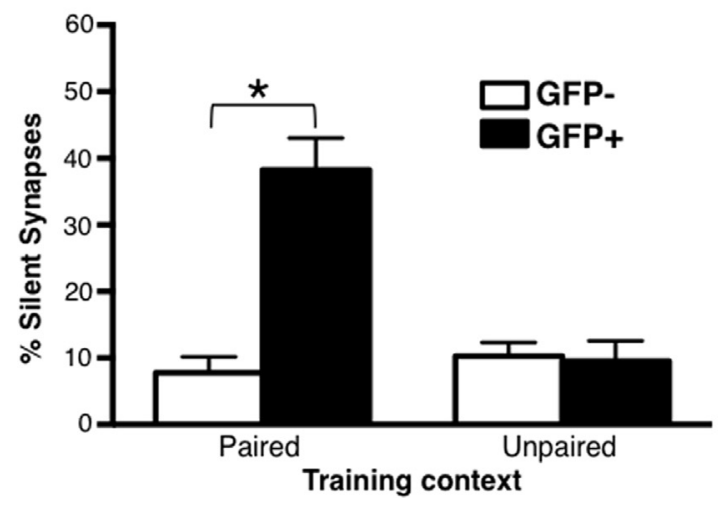

Figure 5. Synaptic alterations in green fluorescent protein-positive $(\mathrm{GFP}+)$ and green fluorescent protein-negative (GFP-) neurons after context-specific sensitization. (A) Double-labeling for Fos and neuronal nuclear antigen (NeuN) in the nucleus accumbens shell after context-specific sensitization. Fos-labeled neurons are shown in green (left), NeuNlabeled neurons are shown in red (middle), and Fos + NeuN doublelabeled neurons are shown in yellow (right). Cocaine induced Fos in $4.79 \%$ of NeuN-labeled neurons after context-specific sensitization in mice in the paired group. Scale bar $=50 \mu \mathrm{m}$. (B) Representative minimal stimulation assays of GFP+ neurons from mice in the paired and unpaired groups. Dots in each graph represent consecutive excitatory postsynaptic currents (EPSC) from a single GFP+ neuron. Representative EPSC traces are shown to the right of the graphs. The top trace was recorded at a holding potential of $+40 \mathrm{mV} \quad(\mathrm{N}$-methyl-D-aspartate receptor- and alpha-amino-3-hydroxy5-methyl-4-isoxazole propionic acid receptor-mediated current). The middle trace (purple) is an example of a synaptic failure. The bottom trace was recorded at a holding potential of -80 $\mathrm{mV}$ (alpha-amino-3-hydroxy-5-methyl4-isoxazole propionic acid receptormediated current). (C) Percentage of silent synapses after context-specific sensitization ( $n=7-8$ cells from $5-7$ mice/group). Silent synapses were more frequent in GFP + neurons $(* p<$ .0001) of mice in the paired group, but not mice in the unpaired group. play causal roles in the expression of context-specific reinstatement of heroin and cocaine seeking, respectively $(1,2)$ as well as neuronal ensembles in orbitofrontal cortex in incubation of cue-induced heroin seeking (3). Based on these studies, silent synapses appear to be induced in Fosexpressing ensembles that mediate expression of the learned association between the drug-paired context and cocaine. This idea is further supported by the finding that repeated cocaine injections, but not a single cocaine injection, in the same context are required to induce silent synapses in Fosexpressing ensembles (20). Overall, our findings indicate that silent synapses are induced only in neurons that were repeatedly activated during training and then reactivated on test day in the same context.

Global alterations of silent synapses and other synaptic alterations, such as AMPA $/ N$-methyl-D-aspartate ratio (9),
AMPA receptor long-term depression (31), and A-type potassium currents (32), have also been reported after cocaine sensitization. However, these global alterations do not have the required degree of resolution to encode the high-resolution information that defines the different contexts in contextspecific sensitization. These global alterations instead may play important roles in preparing neurons for learning-specific alterations or generally increasing or decreasing the expression of the learned behaviors.

The detailed mechanism underlying the formation of silent synapses in these Fos-expressing and GFP-expressing neurons is unknown. Previous studies demonstrated increased silent synapse formation in the general population of nucleus accumbens neurons after cocaine locomotor sensitization $(15,16)$. In these studies, silent synapses were increased in randomly selected neurons of the nucleus accumbens shell for 


\section{Context-specific sensitization}

A

GFP-

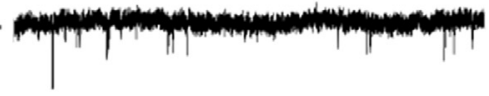

GFP+

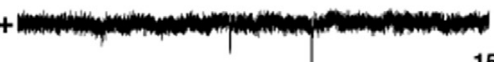

$15 \mathrm{pAL}$

B

Spontaneous EPSC Frequency

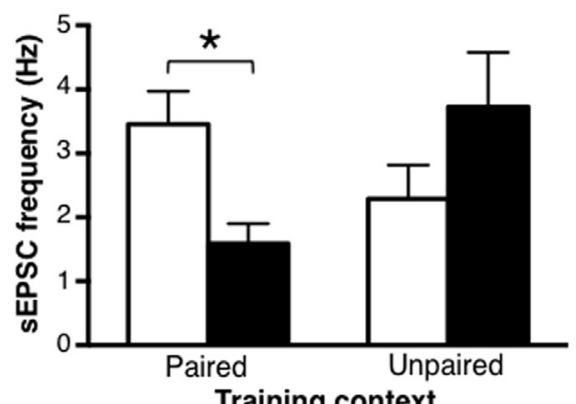

C

Paired: sEPSC Frequency

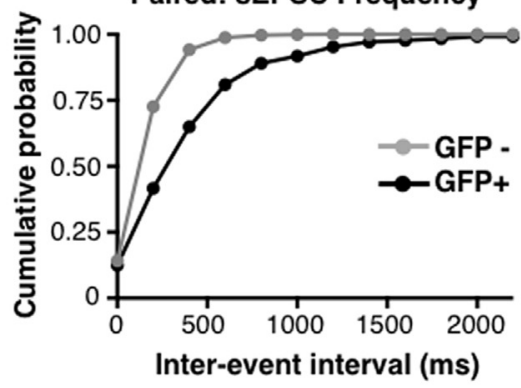

Unpaired: sEPSC Frequency

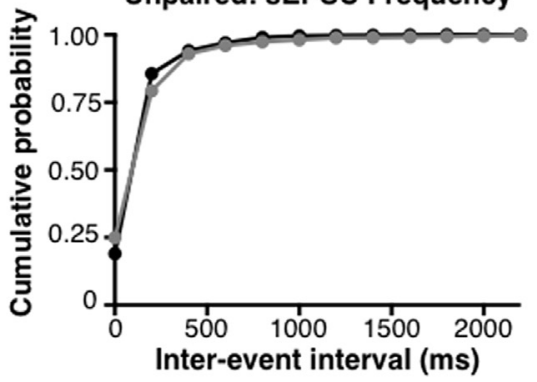

3-5 days after cocaine sensitization as a result of the insertion of new NR2B-containing spines. In contrast, the appearance of new NR2B-containing spines does not underlie the formation of silent synapses that we observed in GFP+ neurons after 6-11 days of withdrawal from cocaine sensitization (20). A possible alternative explanation is that silent synapses in GFP + neurons after context-specific sensitization may involve removal of AMPA receptors from dendritic spines (10, 33-35). It was previously shown that the ratio of surface to intracellular glutamate receptor type 1 subunit is decreased 24 hours after cocaine challenge in sensitized animals, lending support to this idea (36).

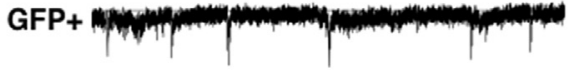

\section{Unpaired}

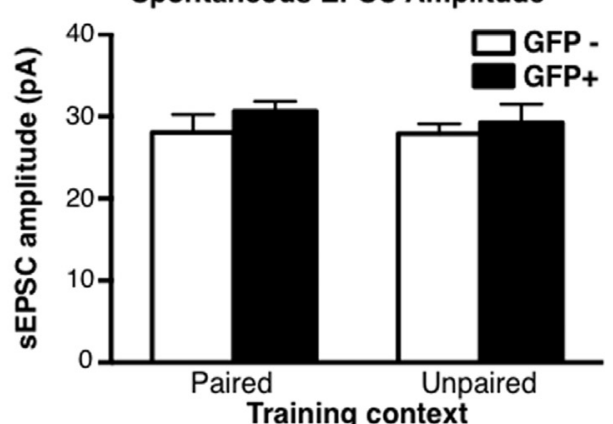

Paired: sEPSC Amplitude

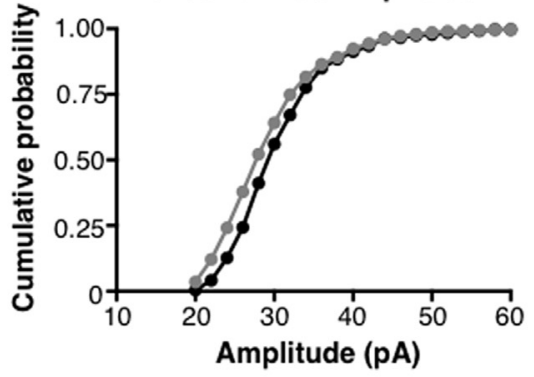

Unpaired sEPSC Amplitude

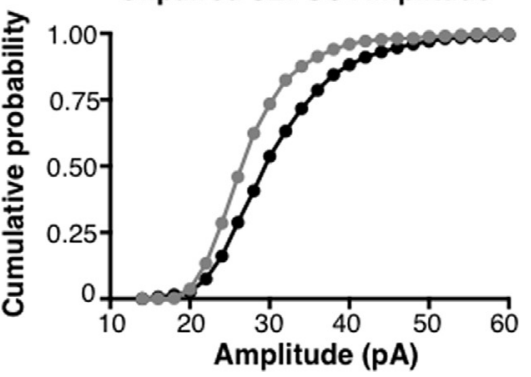

Figure 6. Quantal properties of spontaneous excitatory postsynaptic currents (sEPSCs) after context-specific sensitization. (A) Representative traces showing spontaneous activity in green fluorescent protein-positive (GFP+) and green fluorescent protein-negative (GFP-) neurons from mice in the paired and unpaired groups. (B) sEPSC frequency (left) was significantly lower in GFP+ neurons (paired group, $n=9$ cells from 7 animals; unpaired group, $n=8$ cells from 7 animals) compared with GFPneurons (paired group, $n=8$ cells from 6 animals; unpaired group, $n=$ 12 cells from 10 animals) in the paired group $\left({ }^{*} p<.001\right)$, but not in the unpaired group. SEPSC amplitudes (right) did not differ between groups. (C) Cumulative probability distributions of the same SEPSC frequencies and amplitudes shown in (B)
The appearance of silent synapses exclusively in this activated neuronal population-only in mice that learned the context-reward association-suggests that silent synapses could represent a mechanism for encoding learned associations. However, silent synapses may be induced in strongly activated neurons only as a compensatory mechanism. Strong activation of GFP+ neurons by convergent glutamatergic input as well as dopaminergic modulation $(37,38)$ could cause activated cells to tone down their response by internalizing AMPA receptors (39). In this case, the functional alterations that encode learned associations could precede the development of silent synapses. One prior study determined that there 
was no relationship between AMPA receptor surface expression in the nucleus accumbens and the expression of cocaine sensitization (40). Silent synapses could potentially represent a metaplastic mechanism $(41,42)$ through which synapses may be primed for subsequent experience-induced plasticity, most likely through AMPA receptor insertion. Other metaplastic mechanisms were identified previously $(43,44)$ in several brain regions, which may be modulated by drug exposure $(45,46)$. There is evidence in support of this scenario from studies of the neural correlates of incubation of craving. Rats allowed to self-administer cocaine show an increase in silent synapses on withdrawal day 10 , but by day 45 of withdrawal, when animals are showing increased cue-induced cocaine seeking, synapses are unsilenced by the insertion of $\mathrm{Ca}^{2+}$-permeable AMPA receptors $(34,35)$. Reversal of silent synapse maturation decreases cue-induced craving; however, the question remains as to the specific contribution of silent synapses.

In conclusion, until recently, most studies examined the role of randomly selected neurons in a particular brain area or restricted their focus to a certain cell type because it was impossible to identify the critical population of neurons that encode learned associations (6). Using Fos as an indicator of strong neuronal activation has proven to be reliable in identifying behaviorally activated ensembles that play a causal role in the expression of drug-related learned behavior (4). As mentioned previously, there is a high degree of overlap between the neuronal ensemble that is activated during training and during testing in the animals that express sensitization. One limitation is the need for acute administration of cocaine in the test context on test day to reactivate this population of neurons. A second limitation is the temporal separation between the induction of the Fos promoter and the expression of GFP, which is maximal at 90-120 minutes after induction. Future studies will employ the Fos-Tet-Cre transgenic rat system to identify Fos-expressing neuronal ensembles without the need for acute administration of cocaine to induce Fos and will allow us to follow the time course of the development of silent synapses and determine if silent synapses play a causal role in context-specific sensitization (6).

\section{ACKNOWLEDGMENTS AND DISCLOSURES}

This work was supported by the National Institutes of Health National Institute on Drug Abuse Intramural Research Program and a Capes fellowship from Brazil (PECO).

Experiments were planned by $\mathrm{LRW}, \mathrm{AB}$, and $\mathrm{BTH}$ and carried out by LRW, PECO, KBM, and RVF. LRW, AB, and BTH wrote the manuscript.

We thank Yavin Shaham for helpful suggestions regarding behavioral procedures, statistics, and the manuscript; Jennifer Bossert for assistance with immunohistochemical procedures and statistics; Brandon Warren for assistance with animal perfusions; and Fabio Cruz, Rodrigo Leao, and Klil Babin for assistance in running behavioral experiments.

The authors report no biomedical financial interests or potential conflicts of interest.

\section{ARTICLE INFORMATION}

From the Behavioral Neuroscience Branch (LRW, KBM, RVF, AB, BTH), National Institutes of Health National Institute on Drug Abuse Intramural Research Program, Baltimore, Maryland; and Laboratório de Neuropsicofarmacologia (PECO, CSP), PANT, Faculdade de Ciências Farmacêuticas, Universidade Estadual Paulista, Araraquara, San Paulo, Brazil.
Address correspondence to Bruce T. Hope, Ph.D., NIDA Intramural Research Program, 251 Bayview Boulevard, Baltimore, MD 21224; E-mail: bhope@intra.nida.nih.gov.

Received Jun 18, 2015; revised Aug 5, 2015; accepted Aug 6, 2015.

Supplementary material cited in this article is available online at http://dx.doi.org/10.1016/j.biopsych.2015.08.006.

\section{REFERENCES}

1. Bossert JM, Stern AL, Theberge FR, Cifani C, Koya E, Hope BT, et al. (2011): Ventral medial prefrontal cortex neuronal ensembles mediate context-induced relapse to heroin. Nat Neurosci 14:420-422.

2. Cruz FC, Babin KR, Leao RM, Goldart EM, Bossert JM, Shaham Y, et al. (2014): Role of nucleus accumbens shell neuronal ensembles in contextinduced reinstatement of cocaine-seeking. J Neurosci 34:7437-7446.

3. Fanous S, Goldart EM, Theberge FR, Bossert JM, Shaham Y, Hope BT (2012): Role of orbitofrontal cortex neuronal ensembles in the expression of incubation of heroin craving. J Neurosci 32:11600-11609

4. Koya E, Golden SA, Harvey BK, Guez-Barber DH, Berkow A, Simmons DE, et al. (2009): Targeted disruption of cocaine-activated nucleus accumbens neurons prevents context-specific sensitization. Nat Neurosci 12:1069-1073

5. Cruz FC, Rubio FJ, Hope BT (2015): Using c-fos to study neuronal ensembles in corticostriatal circuitry of addiction. Brain Res 35: 5625-5639.

6. Cruz FC, Koya E, Guez-Barber DH, Bossert JM, Lupica CR, Shaham Y, et al. (2013): New technologies for examining the role of neuronal ensembles in drug addiction and fear. Nat Rev Neurosci 14:743-754.

7. Ungless MA, Whistler JL, Malenka RC, Bonci A (2001): Single cocaine exposure in vivo induces long-term potentiation in dopamine neurons. Nature 411:583-587

8. Borgland SL, Malenka RC, Bonci A (2004): Acute and chronic cocaine-induced potentiation of synaptic strength in the ventral tegmental area: Electrophysiological and behavioral correlates in individual rats. J Neurosci 24:7482-7490.

9. Kourrich S, Rothwell PE, Klug JR, Thomas MJ (2007): Cocaine experience controls bidirectional synaptic plasticity in the nucleus accumbens. J Neurosci 27:7921-7928.

10. Wolf ME, Ferrario CR (2010): AMPA receptor plasticity in the nucleus accumbens after repeated exposure to cocaine. Neurosci Biobehav Rev 35:185-211.

11. Mameli M, Bellone C, Brown MT, Luscher C (2011): Cocaine inverts rules for synaptic plasticity of glutamate transmission in the ventral tegmental area. Nat Neurosci 14:414-416.

12. Mameli M, Balland B, Lujan R, Luscher C (2007): Rapid synthesis and synaptic insertion of GluR2 for mGluR-LTD in the ventral tegmental area. Science 317:530-533.

13. Conrad KL, Tseng KY, Uejima JL, Reimers JM, Heng LJ, Shaham Y, et al. (2008): Formation of accumbens GluR2-lacking AMPA receptors mediates incubation of cocaine craving. Nature 454:118-121.

14. Fourgeaud L, Mato S, Bouchet D, Hemar A, Worley PF, Manzoni OJ (2004): A single in vivo exposure to cocaine abolishes endocannabinoidmediated long-term depression in the nucleus accumbens. J Neurosci 24:6939-6945.

15. Brown TE, Lee BR, Mu P, Ferguson D, Dietz D, Ohnishi YN, et al. (2011): A silent synapse-based mechanism for cocaine-induced locomotor sensitization. J Neurosci 31:8163-8174.

16. Huang YH, Lin Y, Mu P, Lee BR, Brown TE, Wayman G, et al. (2009): In vivo cocaine experience generates silent synapses. Neuron 63:40-47.

17. Pascoli V, Turiault M, Luscher C (2012): Reversal of cocaine-evoked synaptic potentiation resets drug-induced adaptive behaviour. Nature 481:71-75.

18. Whitaker LR, Degoulet M, Morikawa H (2013): Social deprivation enhances VTA synaptic plasticity and drug-induced contextual learning. Neuron 77:335-345.

19. Chen BT, Bowers MS, Martin M, Hopf FW, Guillory AM, Carelli RM, et al (2008): Cocaine but not natural reward self-administration nor passive cocaine infusion produces persistent LTP in the VTA. Neuron 59:288-297. 
20. Koya E, Cruz FC, Ator R, Golden SA, Hoffman AF, Lupica CR, et al. (2012): Silent synapses in selectively activated nucleus accumbens neurons following cocaine sensitization. Nat Neurosci 15:1556-1562.

21. Vanderschuren LJ, Kalivas PW (2000): Alterations in dopaminergic and glutamatergic transmission in the induction and expression of behavioral sensitization: A critical review of preclinical studies. Psychopharmacology 151:99-120.

22. Pierce RC, Kalivas PW (1995): Amphetamine produces sensitized increases in locomotion and extracellular dopamine preferentially in the nucleus accumbens shell of rats administered repeated cocaine. J Pharmacol Exp Ther 275:1019-1029.

23. Vezina P, Leyton M (2009): Conditioned cues and the expression of stimulant sensitization in animals and humans. Neuropharmacology 56(suppl 1):160-168.

24. Badiani A, Robinson TE (2004): Drug-induced neurobehavioral plasticity: The role of environmental context. Behav Pharmacol 15:327-339.

25. Robinson TE, Browman KE, Crombag HS, Badiani A (1998): Modulation of the induction or expression of psychostimulant sensitization by the circumstances surrounding drug administration. Neurosci Biobehav Rev 22:347-354.

26. Stewart J, Badiani A (1993): Tolerance and sensitization to the behavioral effects of drugs. Behav Pharmacol 4:289-312.

27. Carelli RM, Deadwyler SA (1996): Dose-dependent transitions in nucleus accumbens cell firing and behavioral responding during cocaine self-administration sessions in rats. J Pharmacol Exp Ther 277:385-393.

28. Carelli RM, King VC, Hampson RE, Deadwyler SA (1993): Firing patterns of nucleus accumbens neurons during cocaine self-administration in rats. Brain Res 626:14-22.

29. Hebb D (1949): The Organization of Behavior, a Neuropsychological Theory. New York: Wiley Subscription Services, Inc., A Wiley Company.

30. Mattson BJ, Koya E, Simmons DE, Mitchell TB, Berkow A, Crombag HS, et al. (2008): Context-specific sensitization of cocaine-induced locomotor activity and associated neuronal ensembles in rat nucleus accumbens. Eur J Neurosci 27:202-212.

31. Thomas MJ, Beurrier C, Bonci A, Malenka RC (2001): Long-term depression in the nucleus accumbens: A neural correlate of behavioral sensitization to cocaine. Nat Neurosci 4:1217-1223.

32. Kourrich S, Klug JR, Mayford M, Thomas MJ (2012): AMPARindependent effect of striatal alphaCaMKII promotes the sensitization of cocaine reward. J Neurosci 32:6578-6586.

33. Dalton GL, Wang YT, Floresco SB, Phillips AG (2008): Disruption of AMPA receptor endocytosis impairs the extinction, but not acquisition of learned fear. Neuropsychopharmacology 33:2416-2426.
34. Lee BR, Ma YY, Huang $Y H$, Wang X, Otaka M, Ishikawa M, et al. (2013): Maturation of silent synapses in amygdala-accumbens projection contributes to incubation of cocaine craving. Nat Neurosci 16: 1644-1651.

35. Ma YY, Lee BR, Wang X, Guo C, Liu L, Cui R, et al. (2014) Bidirectional modulation of incubation of cocaine craving by silent synapse-based remodeling of prefrontal cortex to accumbens projections. Neuron 83:1453-1467.

36. Boudreau AC, Reimers JM, Milovanovic M, Wolf ME (2007): Cell surface AMPA receptors in the rat nucleus accumbens increase during cocaine withdrawal but internalize after cocaine challenge in association with altered activation of mitogen-activated protein kinases. J Neurosci 27:10621-10635.

37. Day JJ, Roitman MF, Wightman RM, Carelli RM (2007): Associative learning mediates dynamic shifts in dopamine signaling in the nucleus accumbens. Nat Neurosci 10:1020-1028.

38. Goto Y, Grace AA (2005): Dopamine-dependent interactions between limbic and prefrontal cortical plasticity in the nucleus accumbens: Disruption by cocaine sensitization. Neuron 47:255-266.

39. Bachtell RK, Self DW (2008): Renewed cocaine exposure produces transient alterations in nucleus accumbens AMPA receptor-mediated behavior. J Neurosci 28:12808-12814.

40. Ferrario CR, Li X, Wang X, Reimers JM, Uejima JL, Wolf ME (2010): The role of glutamate receptor redistribution in locomotor sensitization to cocaine. Neuropsychopharmacology 35:818-833.

41. Abraham WC, Bear MF (1996): Metaplasticity: The plasticity of synaptic plasticity. Trends Neurosci 19:126-130.

42. Lee BR, Dong Y (2011): Cocaine-induced metaplasticity in the nucleus accumbens: Silent synapse and beyond. Neuropharmacology 61: 1060-1069.

43. Harnett MT, Bernier BE, Ahn K-C, Morikawa H (2009): Burst-timingdependent plasticity of NMDA receptor-mediated transmission in midbrain dopamine neurons. Neuron 62:826-838.

44. Hunt DL, Puente N, Grandes P, Castillo PE (2013): Bidirectional NMDA receptor plasticity controls CA3 output and heterosynaptic metaplasticity. Nat Neurosci 16:1049-1059.

45. Ahn K-C, Bernier BE, Harnett MT, Morikawa H (2010): IP3 receptor sensitization during in vivo amphetamine experience enhances NMDA receptor plasticity in dopamine neurons of the ventral tegmental area. J Neurosci, 30. ), 6689-6699.

46. Bernier BE, Whitaker LR, Morikawa H (2011): Previous ethanol experience enhances synaptic plasticity of NMDA receptors in the ventral tegmental area. J Neurosci 31:5205-5212. 